\title{
Autonomous EBSD Pattern Classification Performance with Changing Acquisition Parameters
}

Kevin Kaufmann and Kenneth Vecchio

UC SAN DIEGO, La Jolla, California, United States

The recent increase in access to compute resources and algorithmic improvements are motivating significant research into artificial intelligence (AI) applied to automating and improving material analysis. Deep learning, a subset of AI, allows computer systems to autonomously learn patterns in data and construct efficient decision rules for tasks including classification, regression, or segmentation. In material analysis, these tools have largely been applied to techniques requiring analysis of data collected in the form of images [1,2]. Electron backscatter diffraction (EBSD) is one such technique benefitting from these recent efforts to improve material analysis by leveraging deep neural networks [3-7]. EBSD is an SEM-based technique involving the capture of 2D diffraction patterns from the surface of a well-polished sample [8]. Within the last decade, advancements in EBSD equipment have enabled the capture of high definition diffraction patterns at rates exceeding $3,000 \mathrm{~Hz}$ [9]. This creates significant opportunities for increasing the amount of information that can be ascertained from a sample, as well as opens the door for training data intensive deep neural networks.

Deep neural network-based classification of the diffraction patterns is motivated by Hough-based EBSD's susceptibility to structural misclassification; a failure mode that modern EBSD can encounter even when the researcher has complete knowledge of the sample prior to beginning analysis. While several methods to improve phase-differentiation have been proposed, each still requires pre-selection of phases and additional data (e.g. chemistry or simulated diffraction patterns) to be available. In contrast, deep neural network-based methods have demonstrated effective phase differentiation [5] and identification of phases to the space group level [4] without the need for further information. The deep learning approach to EBSD diffraction pattern analysis is capable of these more advanced analyses because it uses all information in the image when assessing a diffraction pattern, whereas traditional Hough-based EBSD pattern analysis discards a significant amount of information.

To promote adoption of these tools, it must be demonstrated that the deep neural network-based approach is not prone to error with changes in the acquisition parameters. Moreover, knowledge of the conditions for which a model fails can guide the future collection of labeled training data to improve subsequent versions. In this work, the deep neural network model (i.e., a convolutional neural network) is trained using diffraction patterns captured with a fixed geometry and SEM settings, and a parametric study is performed to develop an understanding of model performance as several of the most common EBSD operating 
conditions are varied. The specific parameters are frame averaging, detector tilt, detector distance, accelerating voltage, and pattern resolution. With regard to parameters that directly affect the time to collect each pattern and therefore complete a map, such as frame averaging and pattern resolution, the ability to collect the data more rapidly without a significant reduction in performance is of interest. With respect to parameters such as detector tilt, it is important to determine if the model is susceptible to minor or major changes from the training conditions. Parameters such as detector distance and accelerating voltage can cause much more dramatic changes to the EBSPs, and it is therefore necessary to assess their influence, and establish suitable limitations to enable this approach. The effect of changing these parameters is tested using new EBSD patterns collected from one material from each of the six space groups in the model and a dual-phase 2205 duplex stainless steel for visual demonstration. Each time one parameter is varied, the EBSPs are re-collected, and the CNN used to reassess the space group identification. Ultimately, the model is found to retain a high classification accuracy even with significant changes to the diffraction conditions and therefore the EBSPs.

K. Kaufmann was supported by the Department of Defense (DoD) through the National Defense Science and Engineering Graduate Fellowship (NDSEG) Program. K. Kaufmann would also like to acknowledge the support of the ARCS Foundation, San Diego Chapter. K. Vecchio would like to acknowledge the financial generosity of the Oerlikon Group in support of his research group.
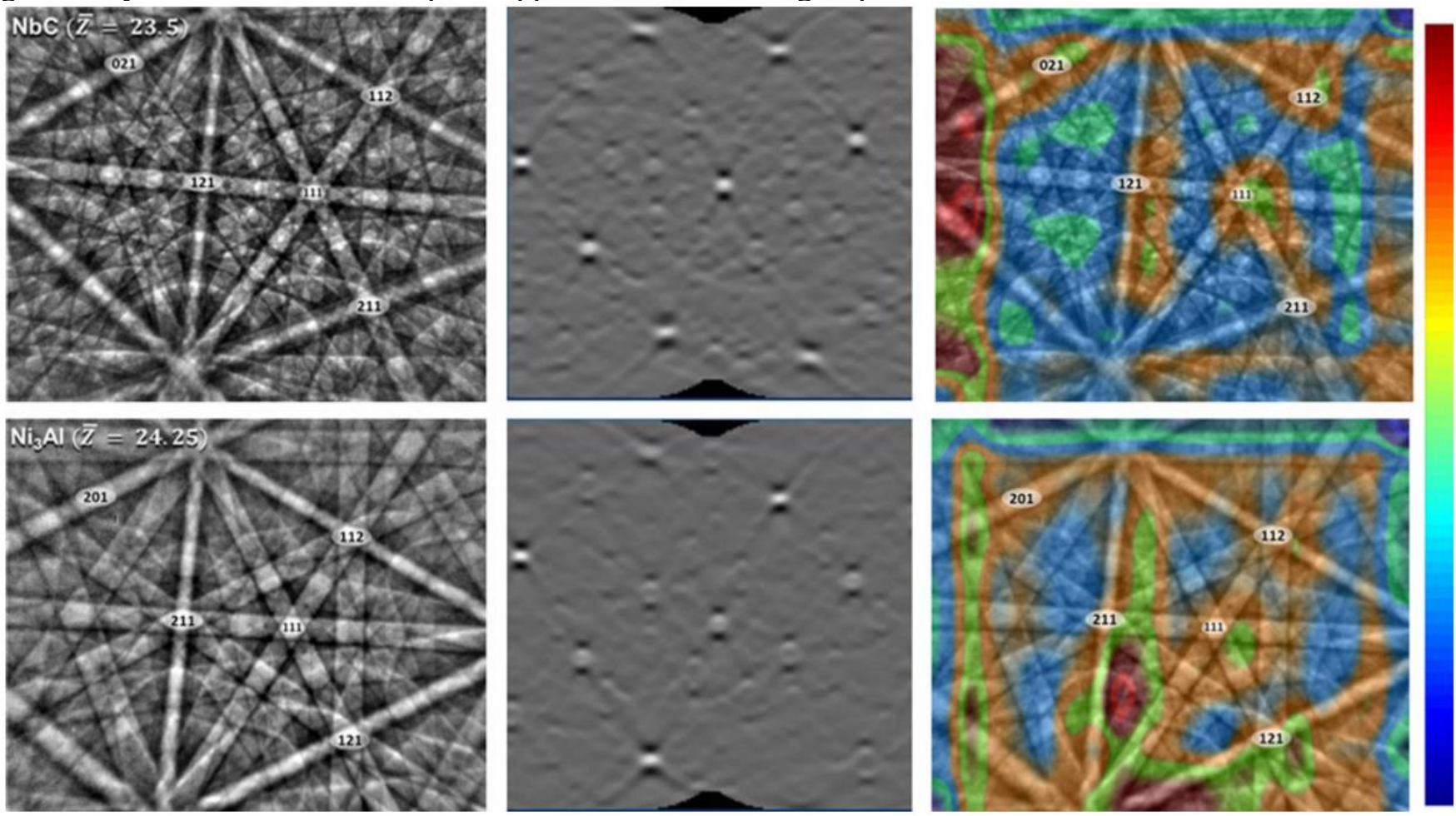

Figure 1. Comparison of feature detection with Hough-based EBSD and the trained CNN. (Top row from left to right) Experimental EBSP from NbC (space group 225; FCC structure), Hough-based feature detection, and gradient-weighted class activated map. (Bottom row from left to right) Experimental EBSP from Ni3Al (space 
group 221; L12 structure), Hough-based feature detection, and gradient-weighted class activated map. The importance scale for the heatmaps goes from dark blue (low) to dark red (high).
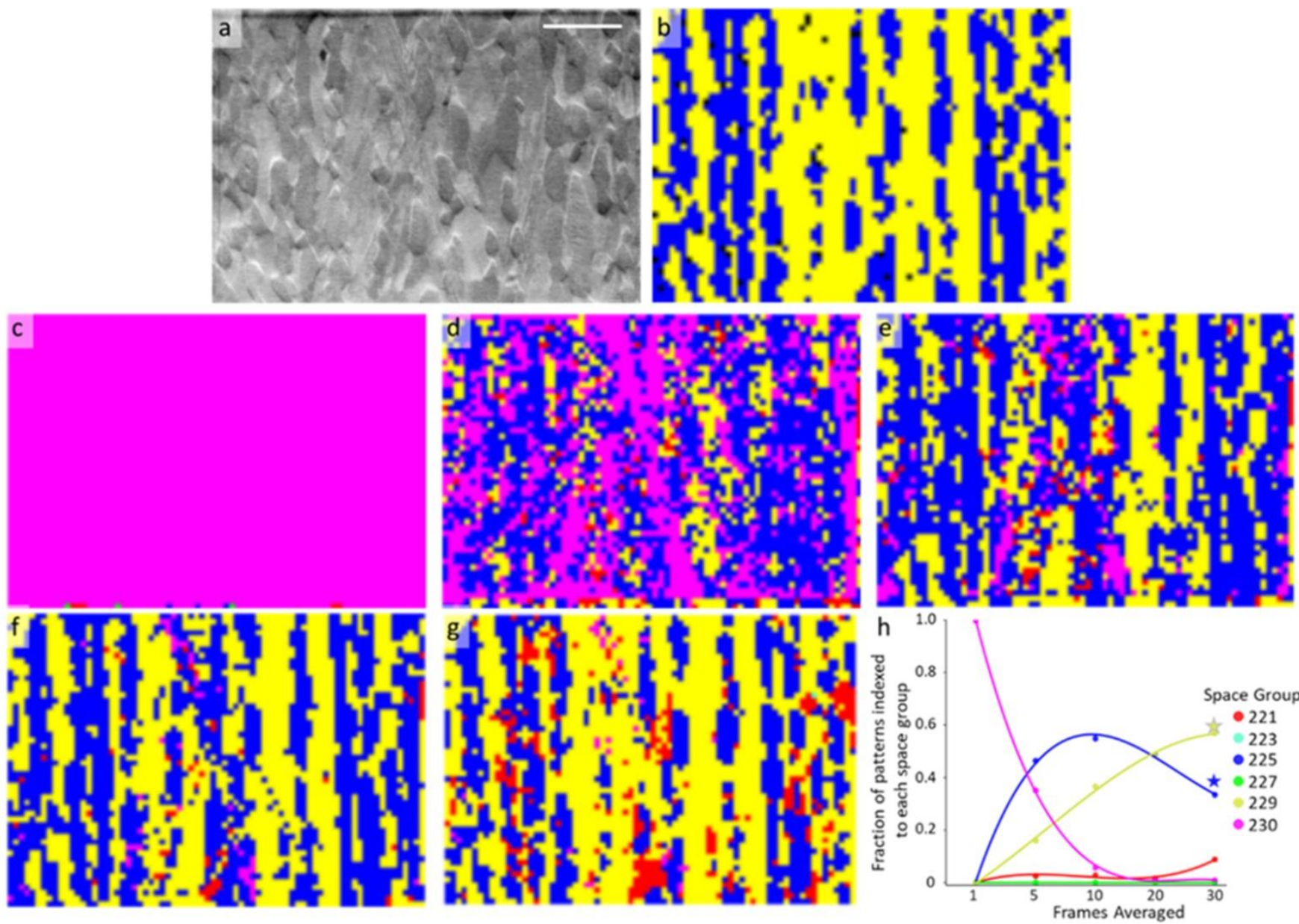

Figure 2. Visual overview of the effect frame averaging during pattern collection has on CNN classifications for duplex steel. (a) electron image of the region of dual-phase 2205 duplex steel. (b) Hough-based EBSD phase map of the fcc (225) austenite (blue) and bcc (229) ferrite (yellow). (c) phase map generated from EBSD patterns collected with no frame averaging applied (i.e. one frame). (d) phase map generated from EBSD patterns collected with five frame averaging applied. (e) phase map generated from EBSD patterns collected with ten frame averaging applied. (f) phase map generated from EBSD patterns collected with twenty frame averaging applied. (g) phase map generated from EBSD patterns collected with thirty frame averaging applied. (h) Plot showing the fraction of patterns indexed to each space group as a function of frame averaging. Thirty frame averaging is the default parameter and is designated as such by the blue star for space group 225 and a yellow star for space group 229. Trend lines are fit with a 3rd order polynomial. Scale bar $25 \mu \mathrm{m}$. There are 3,848 diffraction patterns (pixels) in each phase map.

\section{References}

[1] S.R. Spurgeon, C. Ophus, L. Jones, A. Petford-Long, S. V. Kalinin, M.J. Olszta, R.E. Dunin-Borkowski, N. Salmon, K. Hattar, W.C.D. Yang, R. Sharma, Y. Du, A. Chiaramonti, H. Zheng, E.C. Buck, L. Kovarik, R.L. Penn, D. Li, X. Zhang, M. Murayama, M.L. Taheri, Towards data-driven next-generation transmission electron microscopy, Nat. Mater. (2020). doi:10.1038/s41563-020-00833-z.

[2] M. Ge, F. Su, Z. Zhao, D. Su, Deep learning analysis on microscopic imaging in materials science, Mater. Today Nano. 11 (2020) 100087. doi:10.1016/j.mtnano.2020.100087. 
[3] K. Kaufmann, C. Zhu, A.S. Rosengarten, D. Maryanovsky, T.J. Harrington, E. Marin, K.S. Vecchio, Crystal symmetry determination in electron diffraction using machine learning, Science (80-. ). 367 (2020) 564-568. doi:10.1126/science.aay3062.

[4] K. Kaufmann, C. Zhu, A.S. Rosengarten, K.S. Vecchio, Deep Neural Network Enabled Space Group Identification in EBSD, Microsc. Microanal. 26 (2020) 447-457. doi:10.1017/S1431927620001506.

[5] K. Kaufmann, C. Zhu, A.S. Rosengarten, D. Maryanovsky, H. Wang, K.S. Vecchio, Phase Mapping in EBSD Using Convolutional Neural Networks, Microsc. Microanal. 26 (2020) 458-468. doi:10.1017/S1431927620001488.

[6] Z. Ding, E. Pascal, M. De Graef, Indexing of electron back-scatter diffraction patterns using a convolutional neural network, Acta Mater. 199 (2020) 370-382. doi:10.1016/j.actamat.2020.08.046.

[7] Y.F. Shen, R. Pokharel, T.J. Nizolek, A. Kumar, T. Lookman, Convolutional neural network-based method for real-time orientation indexing of measured electron backscatter diffraction patterns, Acta Mater. 170 (2019) 118-131. doi:10.1016/j.actamat.2019.03.026.

[8] A.J. Schwartz, M. Kumar, B.L. Adams, D.P. Field, Electron backscatter diffraction in materials science, Springer Science+Business Media, LLC, New York, 2009. doi:10.1007/978-0-387-88136-2.

[9] J. Goulden, P. Trimby, A. Bewick, The Benefits and Applications of a CMOS-based EBSD Detector, Microsc. Microanal. 24 (2018) 1128-1129. 